\title{
RED PIGMENT FORMATION BY INTERACTION OF MOLDS

\author{
PART 4 INTERACTION BETWEEN \\ P. VERRUCULOSUM AND VARIOUS MOLDS
}

\author{
SEIICHI NASUNO and TOSHINOBU ASAI \\ The Institute of Applied Microbiology, University of Tokyo \\ Received for publication, Oct. 5, 1961
}

It was reported in previous papers (1-3) that some red pigments were produced by interaction between Penicillium verruculosum and some strains or species of the genera Penicillium and Trichoderma. It was inferred that the pigments are drived from their colorless precursor(s) formed by $P$. verruculosum, which are later converted into the colored material by the action of some other molds. In order to confirm the validity of this inference, further investigations were carried out on the possibility of red pigment formation by several strains of $P$. verruculosum grown in combination with various strains belonging to the genera Aspergillus, Rhizopus, Mucor as well as those belonging to the classes Deuteromycetes (Fungi Imperfecti) and Basidiomycetes.

\section{EXPERIMENTAL METHODS}

1) Microorganisms: The strains tested as possible producers of precursors were strain L 9 isolated by the authors and strains I. A. M. 7058, 7064, 7067, 7073 and 7079 of $P$. verruculosum obtained from the collection at the Institute of Applied Microbiology, University of Tokyo. As secondary molds which are supposed to convert the colorless precursor(s) into the red pigments, tests were made with some representative species of the genera Aspergillus (Table 1), Rhizopus (Table 2) and Mucor (Table 2), obtained from the same collection, and some representative species of the classes Deuteromycetes (Tables 3 and 4) and Basidiomycetes (Table 5) which were provided generously by the Institute of Fermentation, Ministry of International Trade and Industry.

2) Pigment formation: The culture plates were prepared with glucosepeptone agar containing 4.0\% glucose and 1.0\% Difco-peptone, (pH 6.0). Spores of each strain of $P$. verruculosum and spores or mycelial fraction of each strain of the secondary molds were inoculated at a distance of about 3 $\mathrm{cm}$ on the same plate. They were incubated at $30^{\circ}$ for 5 to 7 day, and the formation of the red pigments was observed during the course of incubation 
Table 1. Red pigment formation in co-biontic cultures of several strains of $P$. verruculosum with some species of the genus Aspergillus.

\begin{tabular}{|c|c|c|c|c|c|c|c|c|}
\hline $\begin{array}{l}\text { P. verrucu- } \\
\text { losum } \\
\text { Aspergillus }\end{array}$ & 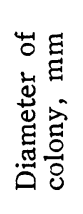 & 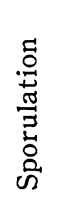 & 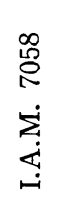 & 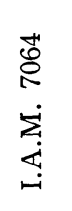 & 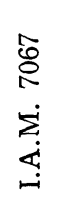 & 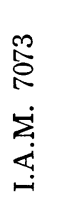 & 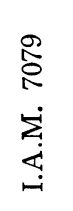 & の \\
\hline A. clavatus & 44 & $(-)$ & $(-)$ & $(-)$ & $(-)$ & $\frac{\text { 册 }}{H}$ & $(-)$ & $(-)$ \\
\hline A. glaucus & 22 & $(-)$ & $(-)$ & $(-)$ & $(-)$ & $\begin{array}{l}\text { H } \\
+-\end{array}$ & $(-)$ & $(-)$ \\
\hline A. fumigatus & 80 & $(-)$ & $(-)$ & $\frac{(-)}{H}$ & $(-)$ & $\frac{H}{H}$ & $(-)$ & $(-)$ \\
\hline A. nidulans & 84 & $(-)$ & $(-)$ & $\frac{(-)}{H}$ & $(-)$ & $\stackrel{+}{H+}$ & $(-)$ & $(-)$ \\
\hline A. flavipes & 86 & + & $(-)$ & $\begin{array}{l}H \\
H\end{array}$ & $\frac{H}{H}$ & $\frac{m}{m}$ & $(-)$ & $\frac{(-)}{H^{*}}$ \\
\hline A. versicolor & 10 & H & $(-)$ & $(-)$ & $\frac{H}{+}$ & $\frac{H}{+}$ & $\frac{+*}{H}$ & $\frac{( \pm)}{t}$ \\
\hline A. niger & 100 & H & $(-)$ & $\frac{t}{(-)}$ & + & $\frac{H}{H}$ & $\frac{(-)}{t}$ & $\frac{H}{H}$ \\
\hline A. tamarii & 84 & + & $\frac{(-)}{H^{*}}$ & $\frac{(-)}{H^{*}}$ & $\begin{array}{l}H \\
H\end{array}$ & $\frac{H}{H^{*}}$ & $\frac{(-)}{H^{*}}$ & $\frac{(-)}{H^{*}}$ \\
\hline A. flavusoryzae & 90 & H & $(-)$ & $\begin{array}{l}+ \\
+\end{array}$ & $\frac{(-)}{H}$ & $\begin{array}{l}\text { H } \\
\text { H }\end{array}$ & $(-)$ & $(-)$ \\
\hline A. ochraceus & 40 & + & $(-)$ & $(-)$ & $(-)$ & $\frac{H}{H}$ & $(-)$ & $(-)$ \\
\hline
\end{tabular}

* : Yellow pigment.

** : Colonies of A. niger overlapped on the colonies of $P$. verruculosum. The marks above and below the bar represents, respectively the degree of pigment formation in $P$. verruculosum and in Aspergillus.

\section{EXPERIMENTAL RESULTS}

1) Pigment formation under interaction with Aspergillus: The results obtained with combined cultures of several strains of $P$. verruculosum with 10 representative species of the genus Aspergillus are preserbed in Table 1. Strain I. A. M. 7073 produced much red pigments, while I. A. M. 7058 and 7079 did not produce the pigments with all the Aspergilli tested. In the combinations of some strains of $P$. verruculosum with A. flavipes, A. tamarii or $A$. versicolor, yellow pigments were produced instead of the red ones. These yellow pigments were generally found along the side of Aspergillus 
Table 2. Red pigment formation by interaction of several strains of $P$. verruculosum with some species of the genus Rhizopus and Mucor.

\begin{tabular}{|c|c|c|c|c|c|c|c|c|}
\hline $\begin{array}{l}\text { P. verru- } \\
\text { culosum } \\
\\
\text { Rhizopus, } \\
\text { Mucor }\end{array}$ & 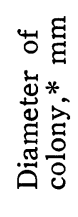 & 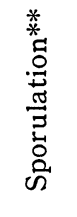 & 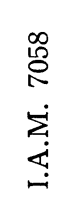 & 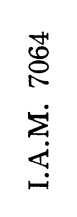 & 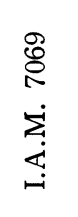 & 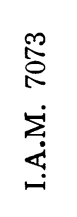 & 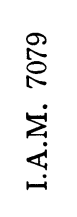 & $\begin{array}{l}\sigma \\
\Delta\end{array}$ \\
\hline R. nigricans & $70>$ & H & $(-)$ & H & $(-)$ & $(-)$ & $(-)$ & $(-)$ \\
\hline R. javanicus & $70>$ & $H$ & $(-)$ & $(-)$ & $(-)$ & $(-)$ & $(-)$ & $(-)$ \\
\hline$R$. chinensis & $70>$ & H & $(-)$ & + & $(-)$ & $(-)$ & $(-)$ & $(-)$ \\
\hline R. arrhizus & $70>$ & $(-)$ & $(-)$ & + & $(-)$ & $(-)$ & $(-)$ & $(-)$ \\
\hline R. salebrosus & $70>$ & H & $(-)$ & + & + & $(-)$ & $(-)$ & $(-)$ \\
\hline R. candidus & $70>$ & $\mathrm{H}$ & $(-)$ & H & + & $(-)$ & $(-)$ & $(-)$ \\
\hline M. spinescens & $70>$ & m & $(-)$ & H & $(-)$ & $(-)$ & $(-)$ & $(-)$ \\
\hline M. racemosus & 32 & $(-)$ & $(-)$ & $(-)$ & $(-)$ & $(-)$ & $(-)$ & $(-)$ \\
\hline M. plumbens & $70>$ & H & $(-)$ & H & + & $(-)$ & $(-)$ & $(-)$ \\
\hline
\end{tabular}

* : Diameter of colony of Rhizopus or Mucor.

** : Sporulation of Rhizopus or Mucor.

colonies except in the combination of $P$. verruculosum with $A$. versicolor. Mycelia of $P$. verruculosum overlapped with those of $A$, niger, but not with those of the other species of Aspergillus. Non-overlapping would be due to some antibiotic substances which were produced and excerted from mycelia of the strains tested. Fig. 1 shows some examples of the red pigment formation occurring between the colonies of $P$. verruculosum and Asper. gillus.

2) Pigment formation under interaction with Rhizopus and Mucor: As shown in Table 2 only two strains of P. verruculosum, i.e. I.A.M. 7064 and 7067, produced the red pigments when grown together with Rhizopus or Mucor. All strains of verruculosum formed no pigment when in contact with R. jaganicus and racemosus. Negative results were also obtained when strain L 9 of $P$. verruculosum was co-cultured with each strain of Rhizopus and Mucor examined.

3) Pigment formation under interaction with Deuteromycetes: Formation of the red pigments occasionally occurred when $P$. verruculosum was grown together with some strains of Deuteromycetes (Tables 3 and 4). Especially, strain I.A.M. 7073 of $P$. verruculosum formed a considerably large amount of the red pigments when grown in combination with various strains of 
'Table 3. Red pigment formation by interaction of several strains of $P$. verruculosum with representative species of Deuteromycetes (I).

\begin{tabular}{|c|c|c|c|c|c|c|c|c|}
\hline $\begin{array}{l}\text { Deutero mycetes } \\
\text { (Fungi Imperfecti) }\end{array}$ & 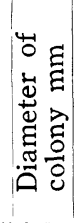 & $\begin{array}{l}0 \\
0 \\
0 \\
0 \\
0 \\
0 \\
0 \\
0 \\
0 \\
0\end{array}$ & 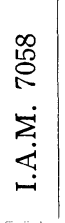 & 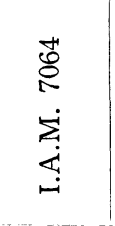 & 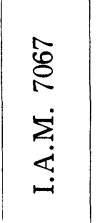 & 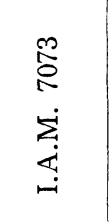 & 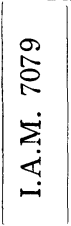 & $\stackrel{\sigma}{\omega}$ \\
\hline \multicolumn{9}{|l|}{ Moniliales } \\
\hline Moniliaceae $\quad$.. & & & & & & & & \\
\hline Acremonium potronii & 15 & - & $(-1)$ & $(-)$ & $(-)$ & $(-)$ & $(-)$ & $(-)$ \\
\hline Acrostalagmus albus & 38 & White & $(-)$ & $H /+$ & $(-)$ & $\mathrm{H} / \mathrm{H}$ & $(-)$ & $(-)$ \\
\hline Beauveria bassiana & 16 & Yellow & $(-)$ & $(-)$ & $(-)$ & $+1 \mathrm{H}$ & $(-)$ & $(-)$ \\
\hline Botrytis reptans & 11 & $\begin{array}{c}\text { Yellowish } \\
\text { green }\end{array}$ & $(-)$ & $(-)$ & $(-)$ & $+1 \mathrm{H}$ & $(-)$ & $(-)$ \\
\hline Candelo spora penialloides & 26 & Yellow & $(-1)$ & $* \# /(-)$ & $(-)$ & $H / H$ & $(-)$ & $(-)$ \\
\hline Corethropsis hominis & 8 & $\begin{array}{c}\text { Greenish } \\
\text { black }\end{array}$ & $(-1)$ & $(-)$ & $(-)$ & $(-)$ & $(-)$ & $(-)$ \\
\hline Gliocladium $s p$ & 40 & White & $(-1)$ & $H / H$ & $*+1+$ & $+/$ H & $(-)$ & $(-)$ \\
\hline Monosporium apiospermum & 30 & White & $(-)$ & $H /(-)$ & $(-)$ & $(-) / H$ & $(-)$ & $(-)$ \\
\hline Mycogone japii & 48 & $\begin{array}{l}\text { Greenish } \\
\text { white }\end{array}$ & $(-1)$ & $H /(-)$ & $(-)$ & $\mathrm{H} / \mathrm{H}$ & $(-)$ & $(-)$ \\
\hline Piricularia oryzae & 26 & White & $(-)$ & $H /+$ & $(-)$ & $\mathrm{m} / \mathrm{m} / \mathrm{m}$ & $(-)$ & $(-)$ \\
\hline Spicaria viridans & 17 & $\begin{array}{c}\text { Yellowish } \\
\text { brown }\end{array}$ & $(-)$ & $(-)$ & $(-)$ & $(-)$ & $(-)$ & $(-)$ \\
\hline Tricothecium roseum & 32 & White & $(-1)$ & $Y+1+$ & $(-)$ & $\mathrm{H} / \mathrm{H}$ & $(-)$ & $\mathrm{Y} / \mathrm{H}(-)$ \\
\hline Tritirachium purpureum & 5 & Yellow & $(-)$ & $(-)$ & $(-)$ & $(-) / H$ & $(-)$ & $(-)$ \\
\hline Vertricillium nieveostratosum & 16 & White & $(-)$ & $(-)$ & $(-)$ & $* H / H$ & $(-)$ & $(-)$ \\
\hline \multicolumn{9}{|l|}{ Stilbaceae } \\
\hline Mycoderma monosum & 7 & Yellow & $(-1)$ & $H /(-)$ & $(-)$ & $+1+$ & $(-)$ & $(-)$ \\
\hline Sporobolomyces roseum & 7 & $\begin{array}{c}\text { Yellowish } \\
\text { orange }\end{array}$ & $(-)$ & $(-)$ & $(-)$ & $(-)$ & $(-)$ & $(-)$ \\
\hline Microsporium andouini & 14 & White & $(-)$ & $(-)$ & $(-)$ & $(-)$ & $(-)$ & $(-)$ \\
\hline Isaria farinosa & 31 & White & $(-)$ & $(-)$ & $(-)$ & $\mathrm{H} / \mathrm{H}$ & $(-)$ & $(-)$ \\
\hline Stysanus medius & 40 & Grey & $(-)$ & $(-)$ & $(-)$ & $+1+$ & $(-)$ & $(-)$ \\
\hline Histoplasma capsulatum & 4 & White & $(-)$ & 冊/H & $+/(-)$ & $H /+$ & $(-)$ & $(-)$ \\
\hline Parendomyces asteroides & 13 & White & $(-)$ & $(-)$ & $(-)$ & $H / H$ & $(-)$ & $(-)$ \\
\hline Pseudomycoderma miso & 11 & White & $(-)$ & $+/(-)$ & $(-)$ & H/W & $(-)$ & $(-)$ \\
\hline \multicolumn{9}{|l|}{ Tuberculariaceae } \\
\hline Epicoccum purpurascens & 60 & $\begin{array}{c}\text { Yellowish } \\
\text { orange }\end{array}$ & $(-)$ & $+/(-)$ & $(-)$ & $+1 H$ & $(-)$ & $(-)$ \\
\hline Fusarium oxysporum & 80 & White & $(-)$ & $\mathrm{H} / \mathrm{W}$ & $+1(-)$ & $\mathrm{H} /+$ & $(-)$ & $(-)$ \\
\hline
\end{tabular}

Note: 1) * : Both colonies were overlapped on each other

2) Y : Yellow pigment, (B : brown pigment)

3) The marks below the bars represent pigment formation in Deuteromycetes colonies.

4) The marks above the bars represent pigment formation in Deuteromycetes colonies.

5) Diameter of colony and reverse side of colony are those of Deuteromycetes. 
Table 4. Red pigment formation by interaction of several strains of $P$. verruculosum with representative species of Deuteromycetes (II).

\begin{tabular}{|c|c|c|c|c|c|c|c|c|}
\hline $\begin{array}{l}\text { Deuteromycetes } \\
\text { (Fungi Imperfecti }\end{array}$ & 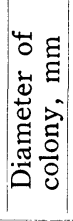 & 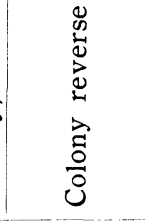 & 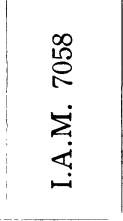 & 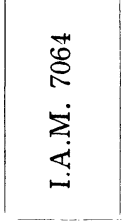 & 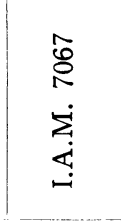 & 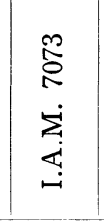 & 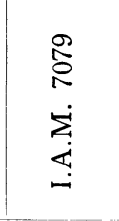 & $\stackrel{\infty}{\oplus}$ \\
\hline \multicolumn{9}{|l|}{ Moniliales } \\
\hline \multicolumn{9}{|l|}{ Dermatiaceae } \\
\hline Cladosporium olivaceum & 92 & $\begin{array}{l}\text { Yellowish } \\
\text { white }\end{array}$ & $\stackrel{*}{(-)} /+\mathrm{Y}$ & $(-) /+B$ & $\stackrel{*}{\mathrm{~B}} \mathrm{H} /+\mathrm{B}$ & $* \mathrm{H} / \mathrm{m}$ m & $* \mathrm{~B}$ \#/H & $\mathrm{B} H / \mathrm{H}$ \\
\hline Altenaria citri & 80 & Black & $(-)$ & $(-)$ & $(-)$ & $* H / m$ & $(-)$ & $(-)$ \\
\hline Cereosporina kikuchi & 24 & $\begin{array}{l}\text { Reddish } \\
\text { purple }\end{array}$ & $(-) /+$ & $(-)$ & $(-)$ & $(-) / H$ & $(-)$ & $(-)$ \\
\hline Cercospora oryzae & 114 & Black & $*(-)$ & $(-)$ & $(-)$ & $(-) / H$ & $(-)$ & $(-)$ \\
\hline Chalara mycederma & 6 & $\begin{array}{c}\text { Yellowish } \\
\text { white }\end{array}$ & $(-)$ & $(-)$ & $*(-)$ & $*+1+t$ & $(-)$ & $*(-)$ \\
\hline Cladosporium herbarum & 12 & $\begin{array}{l}\text { Greenish } \\
\text { white }\end{array}$ & $(-)$ & $(-)$ & $*(-)$ & $(-)$ & $*(-)$ & $*(-)$ \\
\hline Curvularia tetramera & 55 & Black & $(-)$ & $(-)$ & $(-)$ & $(-)$ & $(-)$ & $(-)$ \\
\hline Dematium pullulans & 37 & Black & $*(-)$ & $(-)$ & $(-)$ & $(-)$ & $(-)$ & $(-)$ \\
\hline Fumago sp. & 6 & Black & $(-)$ & $(-)$ & $(-) / H$ & $(-)$ & $(-)$ & $(-)$ \\
\hline Helicocreas oryzae & 2 & Black & $(-)$ & $(-)$ & $(-;)$ & $(-)$ & $(-)$ & $(-)$ \\
\hline Helminthosporium avenae & 36 & White & $(-)$ & $(-)$ & $(-)$ & $(-)$ & $(-)$ & $(-)$ \\
\hline Hemispora rugosa & 16 & White & $(-)$ & $+1(-)$ & $(-)$ & $\mathrm{H} / \mathrm{H}$ & $(-)$ & $(-)$ \\
\hline Humicola grisea & 37 & Black & $(-)$ & $(-)$ & $(-)$ & $+1 H$ & $(-)$ & $(-)$ \\
\hline Macrosporium bataticola & 12 & Black & $(-)$ & $\mathrm{H} /+$ & $+/(-)$ & $(-) /+$ & $(-)$ & $(-)$ \\
\hline Nigrospora oryzae & 90 & $\begin{array}{l}\text { Brownish } \\
\text { black }\end{array}$ & $(-)$ & $(-)$ & $(-)$ & $+1 \mathrm{H}$ & $(-)$ & $(-)$ \\
\hline Phialophora verrucosa & 10 & Black & $(-)$ & $(-)$ & $(-)$ & $(-) / H$ & $(-)$ & $(-)$ \\
\hline Stachybotrys bicolor & 11 & White & $(-)$ & $+/(-)$ & $(-)$ & H/H & $(-)$ & $(-)$ \\
\hline Stemphylium botryosum & 40 & $\begin{array}{c}\text { Brownish } \\
\text { black }\end{array}$ & $(-)$ & $(-)$ & $(-)$ & $+1 H$ & $(-)$ & $(-)$ \\
\hline Thyrospora astragali & 35 & - & $(-)$ & $(-)$ & $(-)$ & $(-)$ & $(-)$ & $(-)$ \\
\hline Torulopsis aeria & 10 & White & $(-)$ & $(-)$ & $(-)$ & 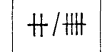 & $(-)$ & $(-)$ \\
\hline \multicolumn{9}{|l|}{ Phomaceae } \\
\hline \multicolumn{9}{|l|}{ Phomaceae } \\
\hline Ascochyta viciae & 5 & 一 & $(-)$ & $(-)$ & $(-)$ & $(-)$ & $(-)$ & $(-)$ \\
\hline Diplodia natalensis & 110 & - & $(-)$ & $(-)$ & $(-)$ & $* H 1+$ & $(-)$ & $(-)$ \\
\hline Phomapsis citri & 2 & - & $(-)$ & $(-)$ & $(-)$ & $(-)$ & $(-)$ & $(-)$ \\
\hline \multicolumn{9}{|l|}{ Melaconiales } \\
\hline Mclaconiaceae & & & & & & & & \\
\hline Gloeosporium laeticolor & 32 & - & $(-)$ & $\mathrm{m} /(-)$ & $(-)$ & $+1 H$ & $(-)$ & $(-)$ \\
\hline Pestalatica diospyri & 26 & - & $(-)$ & $(-) / H$ & $(-)$ & 冊/冊 & $(-)$ & $(-)$ \\
\hline \multicolumn{9}{|l|}{ Ustilaginales } \\
\hline Tilletiaceae & & & & & & & & \\
\hline Tilletiopsis lilacina & 6 & - & $(-)$ & $(--)$ & $(-)$ & $(-)$ & $(-)$ & $(-)$ \\
\hline
\end{tabular}

Note: The same as in Table 3. 
Table 5. Red pigment formation by interaction of several strains of $P$. verruculosum with representative species of Basidiomycetes.

\begin{tabular}{|c|c|c|c|c|c|c|c|c|}
\hline P. verruculosum & 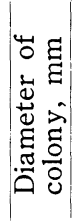 & 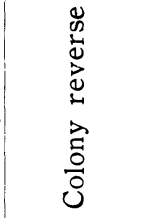 & 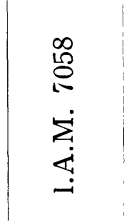 & 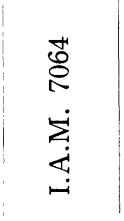 & 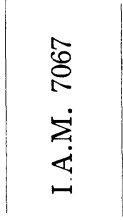 & 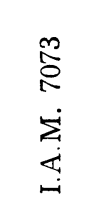 & 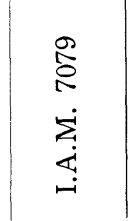 & $\begin{array}{l}\infty \\
\omega\end{array}$ \\
\hline $\begin{array}{l}\text { Agricales } \\
\qquad \text { Agricaceae }\end{array}$ & & & & & & & & \\
\hline Armillaria mellea & 3 & $\begin{array}{l}\text { Yellowish } \\
\text { white }\end{array}$ & $(-)$ & $(-)$ & $(-)$ & $\mathrm{H} / \mathrm{H}$ & $(-)$ & $(-)$ \\
\hline Coprinus comatus & 7 & $\begin{array}{l}\text { Yellowish } \\
\text { white }\end{array}$ & $(-)$ & $(-)$ & $(-)$ & $(-)$ & $(-)$ & $(-)$ \\
\hline Cortinellus edodes & 7 & Brown & $(-)$ & $(-)$ & $(-)$ & $(-) / H$ & $(-)$ & $(-)$ \\
\hline Pholiota nameko & 4 & $\begin{array}{l}\text { Yellowish } \\
\text { white }\end{array}$ & $(-)$ & $*+1+$ & $+/(-)$ & $\mathrm{H} / \mathrm{H}$ & $(-)$ & $(--)$ \\
\hline Pleurotus serotinus & 4 & White & $*(-)$ & $*+1+$ & $(-)$ & $\mathrm{H} / \mathrm{H}$ & $(-)$ & $(-)$ \\
\hline Psalliota campestris & 84 & $\begin{array}{l}\text { Pale } \\
\text { yellow }\end{array}$ & $\mathrm{Y}+1+\mathrm{Y}$ & $(-)$ & m/m & $(-) / H+H$ & $(-) /+\mathrm{Y}$ & $+1 H$ \\
\hline Schizophyllus communs & 52 & $\begin{array}{l}\text { Pale } \\
\text { yellow }\end{array}$ & $(-)$ & $(-)$ & B $+1+\mathrm{B}$ & $+1 \mathrm{H}$ & $(-)$ & $\mathrm{B}^{*}+1+\mathrm{B}$ \\
\hline Tricholoma conglobatum & 60 & White & $*(-)$ & $* H /(-)$ & $(-)$ & $H / H$ & $*(-)$ & $*(-)$ \\
\hline Polyporaceae & & & & & & & & \\
\hline Coniophora puteana & 90 & White & $(-)$ & $*+1+$ & $+1+$ & $\mathrm{H} / \mathrm{H}$ & $(-)$ & $(-)$ \\
\hline Corticium caeruleum & 46 & $\begin{array}{c}\text { Brownish } \\
\text { white }\end{array}$ & $(-)$ & $* H / H$ & $B+/(-)$ & $* \mathrm{H} / \mathrm{H}$ & $\mathrm{B}+/(-)$ & $(-)$ \\
\hline Gloeophyllum sepiarium & 120 & White & $(-)$ & $\mathrm{YH} / \mathrm{H}$ & $\mathrm{YH} /+\mathrm{B}$ & $\mathrm{B}+1 \mathrm{H}$ & $\mathrm{B}+1+\mathrm{B}$ & $\mathrm{B}+\mathrm{H} /+\mathrm{B}$ \\
\hline Lenzites lepideus & 13 & - & $(-)$ & $+1 H$ & $(-)$ & $+1 H$ & $(-)$ & $(-)$ \\
\hline stereum hirsutum & 13 & White & $+1+$ & $(-) / H$ & $(-)$ & $(-) / H$ & $(-) /+$ & \\
\hline Trametes albida & 60 & White & $(-)$ & $(-) / H$ & $(-)$ & $* H / H$ & $\mathrm{~B}+/(-)$ & $\mathrm{BH} /(-)$ \\
\hline Telephoraceae & & & & & & & & \\
\hline Cryptoporus volvatus & - & - & $(-)$ & $(-)$ & $(-)$ & $* H / H$ & $(-)$ & \\
\hline Daedalea quercina & 44 & White & $\left.\mathrm{Y}+/ \mathbf{L}^{-}\right)$ & $\mathrm{H} / \mathrm{H}$ & $\mathrm{B}+1 \mathrm{H}$ & $+/$ m & $\mathrm{B}+/(-)$ & $\mathrm{B}+/(-)$ \\
\hline Favolus arcularius & 60 & White & $\mathrm{Y}+/(-)$ & $* H /(-)$ & $(-)$ & $\mathrm{H} / \mathrm{H}$ & $*(-)$ & $(-)$ \\
\hline Fistulina hepatica & 7 & White & $(-)$ & $(-)$ & $(-)$ & $(-)$ & $(-)$ & \\
\hline Fomes fomentarius & 2 & Yellow & $(-)$ & $(-)$ & $(-)$ & $(-)$ & $(-)$ & $(-)$ \\
\hline Ganoderma lucidum & 44 & $\begin{array}{l}\text { Pale } \\
\text { yellow }\end{array}$ & $(-)$ & $(-)$ & $(-)$ & $\mathrm{Y}+1+$ & $(-)$ & $\mathrm{B}+/(-)$ \\
\hline Merulius confluens & 12 & White & $(-)$ & $(+) /(-)$ & $(-)$ & $+1 H$ & $(-)$ & $(-)$ \\
\hline Polyporus parustris & 66 & White & $(-)$ & $+1+$ & $+1+$ & $H / H$ & $(-)$ & $*+1+$ \\
\hline Poria cocos & 39 & $\begin{array}{c}\text { Brownish } \\
\text { black }\end{array}$ & $(-)$ & $(-)$ & $(-)$ & ${ }^{*} \mathrm{H} / \mathrm{m}$ & $\mathrm{B}+/(-)$ & $(-)$ \\
\hline
\end{tabular}

Note: The same as in Table 3, except Basidiomycetes instead of Deuteromycetes in the note (5). 


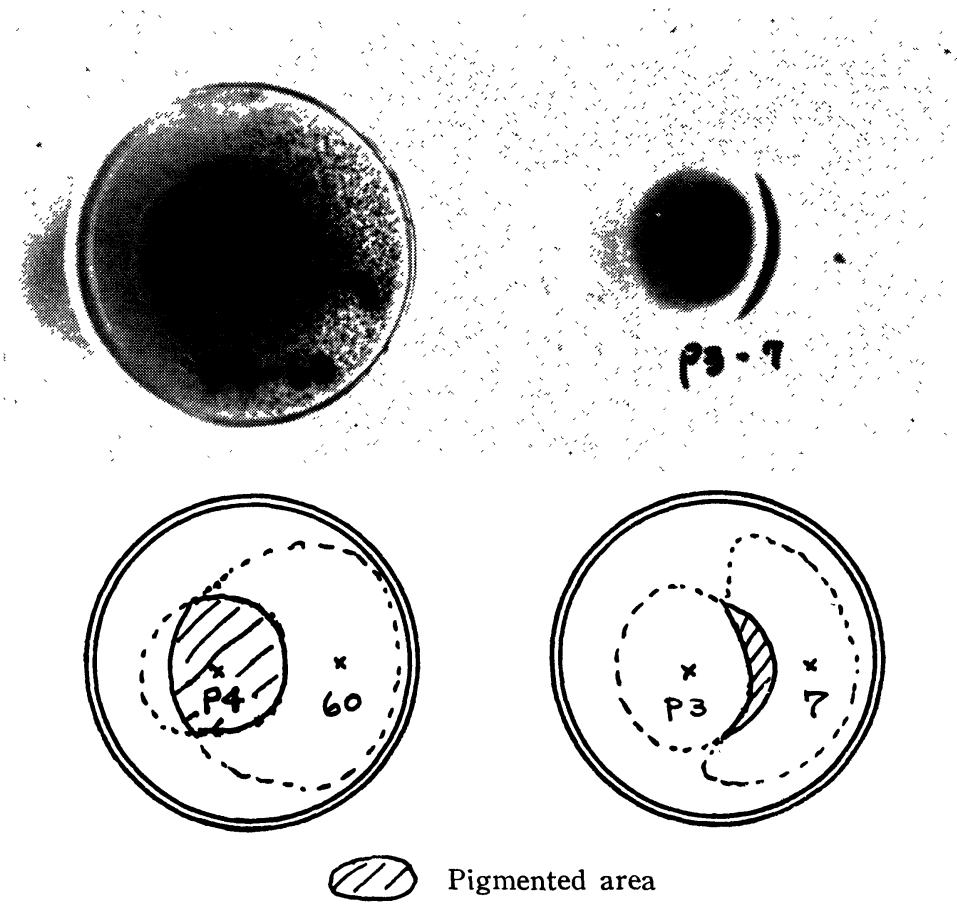

Fig. 1. Red pigment formation by interaction of strains of $P$. verruculosum and those of Aspergillus.

P 4: P. verruculosum I. A. M. 7073

P 3: P. verruculosum I. A. M. 7067

60: A. niger

7: A. tamarii

Deuteromycetes (more than a half of all strains tested). Some pictures of the pigment formation are presented in Fig. 2. In contast, strains I. A. M. 7058, 7067, 7079 and L 9 scarcely produced the red pigments under interaction with most strains of Deuteromycetes.

4) Pigment formation under interaction with Basidiomycetes: Table 5 shows the results obtained when various strains of $P$. verruculosum were cocultured with different species of Basidiomycetes. Some examples of the red pigment formation observed are presented in Fig. 3. In many combinations, strains I.A.M. 7073 and 7064 produced the red pigments remarkably, while strains I.A.M. 7058, 7067, 7079 and L 9 did not do so, although some of them formed yellow and brown pigments. The results in Table 5 show that the formation of yellow and brown pigments is dependent on the strain of Basidiomycetes used for the combination and not on that of $P$. verruculosum. The formation was observed only when a strain of $P$. verruculosum was co-cultured with Gloeophyllum sepiarium, Daedalea quercina, Ganoderma lucidum, Poria cocos and Schizophyllum communs. This observation indicates clearly that the yellow and brown pigments are products 


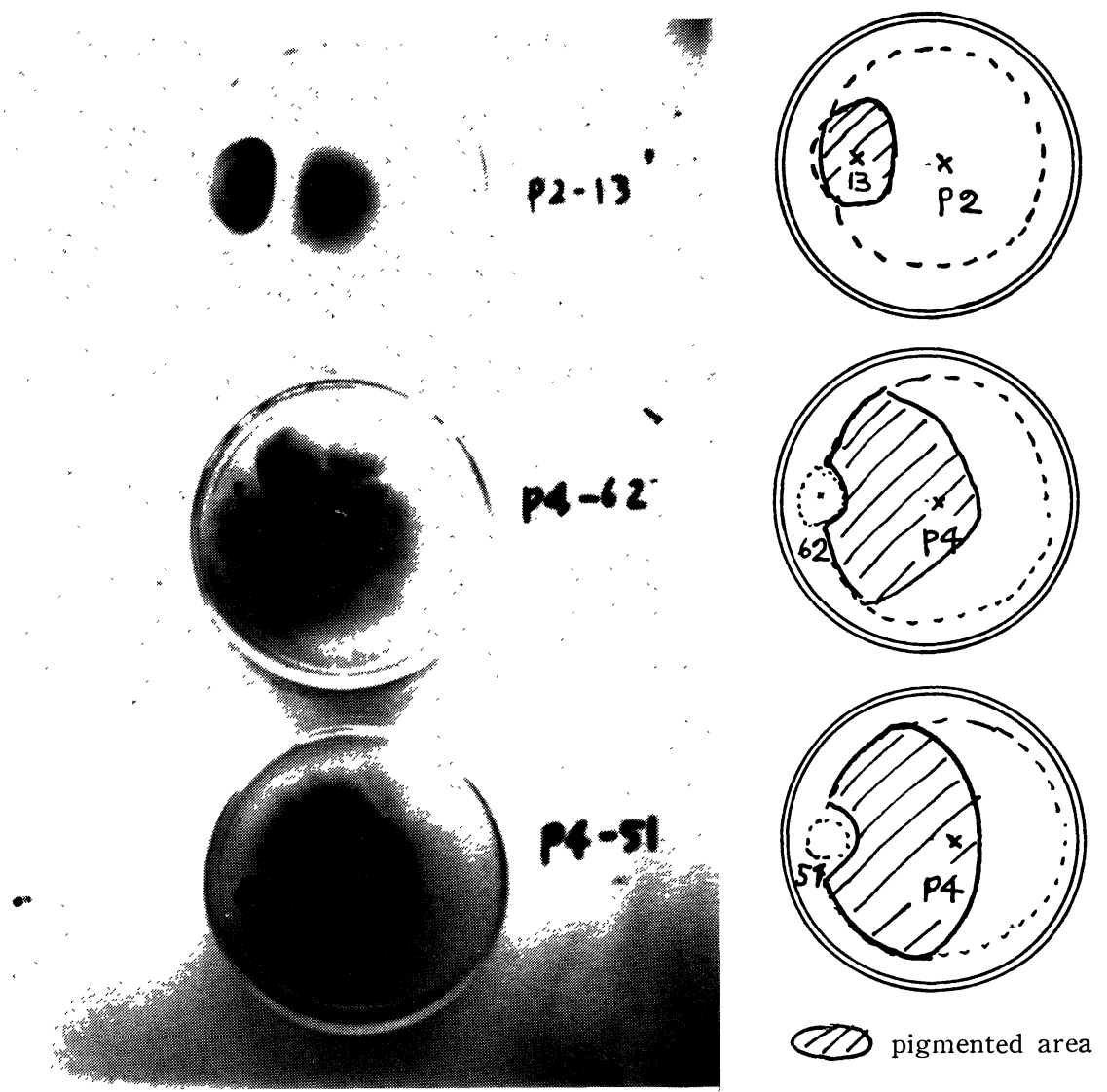

Fig. 2. Red pigment formation by interaction of strains of $P$. verruculosum with those of Deuteromycetes (Fungi Imperfecti).

P 2: P. verruculosum I. A. M. 7064

P 4: P. verruculosum I. A. M. 7073

13: Candelospora penicilloides (Moniliaceae)

62: Hemispora rugosa (Dermatiaceae)

51: Pseudomycoderma miso (Stilbaceae)

primarily formed by Basidiomycetes. Whether these yellow and brown pigments have some relation to the red pigments is a question open to further investigations.

\section{SUMMARY}

Investigations were made on the phenomenon of red pigment formation occurring in co-biontic cultures of Penicillium verruculosum with various other molds belonging to the genera Aspergillus, Rhizopus, Mucor and those 

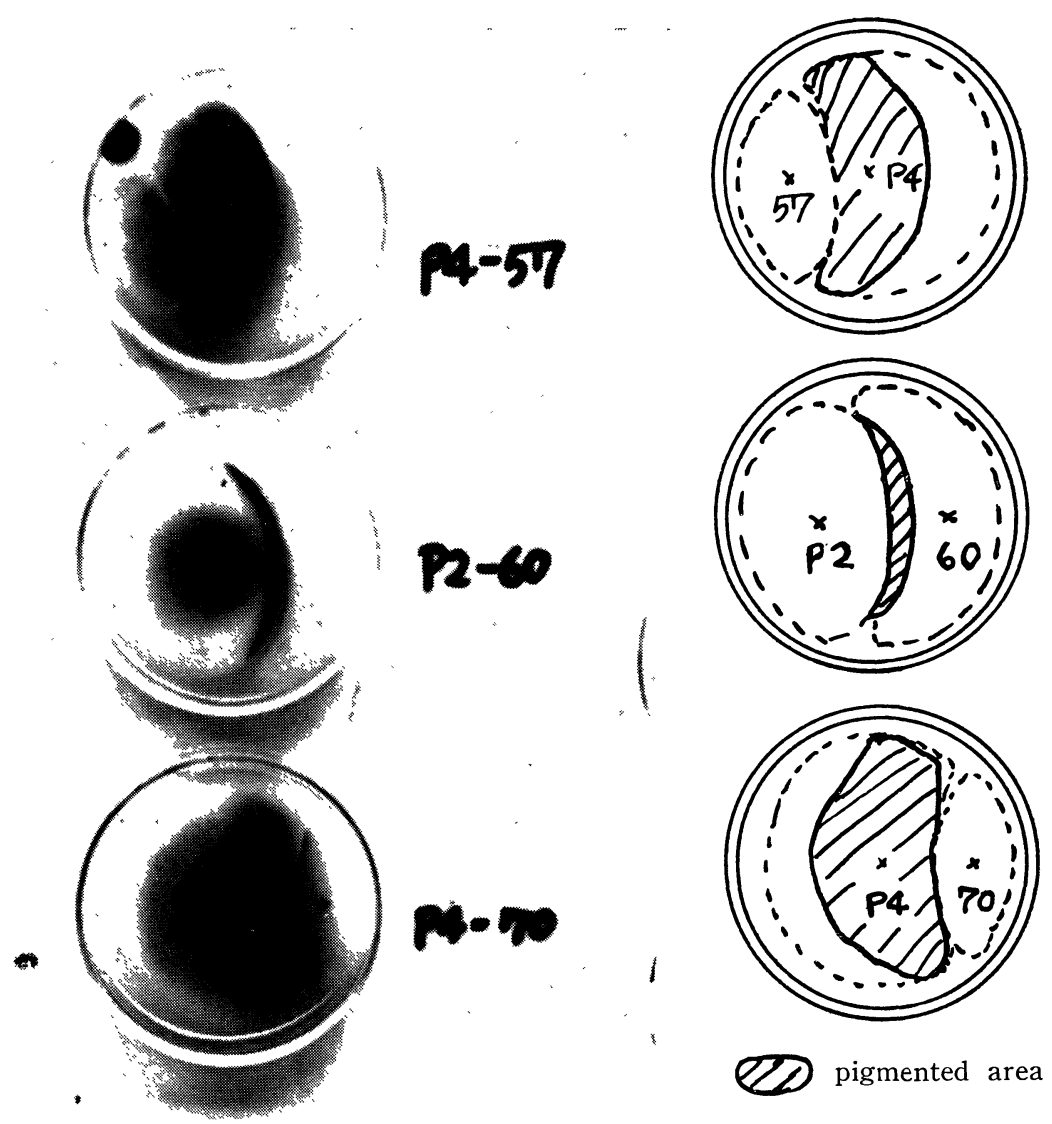

Fig. 3. Red pigment formation by interaction of strains of $P$. verruculosum with those of Basidiomycetes.
P 4: P. verruculosum I. A. M. 7073
P 2: P. verruculosum I. A. M. 7064
57: Psaliota campestris (Agricaceae)
60: Coniophola puteana (Polyporaceae)
70: Daedalea quercina (Telephoraceae)

belonging to the classes Deuteromycetes and Basidiomycetes.

Many species of Aspergillus were found to induce the pigment formation in co-cultures with several strains of P. verruculosum. An exception was Aspergillus tamarii which produced yellow pigment(s) in its mycelia under the same condition. The red pigment formation was seldom observed in the combinations of $P$. verruculosum with some species of Rhizopus and Mucor. It occurred occasionally in the combinations of various species of Deuteromycetes with strain I. A. M. 7073 of $P$. verruculosum, but scarcely with other strains of P. verruculosum. Strains I. A. M. 7064 and 7073 of $P$. verruculosum produced the red pigments when grown together with 
various molds belonging to Basidiomycetes. Some species, especially Glieophyllum sepiarium and Daedalea gercina, produced yellow or brown pigment(s) at the contact margin of their colonies with that of $P$. verruculosum.

Thus, the formation of red pigments by $P$. verruculosum in its cobiontic cultures with other molds was found to be a phenomenon of fairly common occurrence.

\section{ACKNOWLEDGEMENT}

The authors express their thanks to Dr. Akira Kanbayashi, Institute of Fermentation, Ministry of Trade and Industry, for the supply of many cultures of Deuteromycetes and Basidiomycetes.

\section{REFERENCES}

(1) S. NASUNo and T. AsaI: This Journal, 7, 78 (1961)

(2) S. NASUNo and T. AsaI: ibid., 8, 9 (1962)

(3) S. NASUNo and T. ASAI: ibid., 8, 19 (1962) 